Meta

Journal des tradlucteurs

Translators' Journal

\title{
Une oeuvre d'amour et de vertu
}

\section{Roger Duhamel}

Volume 14, numéro 1, mars 1969

URI : https://id.erudit.org/iderudit/002672ar

DOI : https://doi.org/10.7202/002672ar

Aller au sommaire du numéro

Éditeur(s)

Les Presses de l'Université de Montréal

ISSN

0026-0452 (imprimé)

1492-1421 (numérique)

Découvrir la revue

Citer ce document

Duhamel, R. (1969). Une oeuvre d'amour et de vertu. Meta, 14(1), 3-4.

https://doi.org/10.7202/002672ar

Ce document est protégé par la loi sur le droit d'auteur. L'utilisation des services d'Érudit (y compris la reproduction) est assujettie à sa politique d'utilisation que vous pouvez consulter en ligne.

https://apropos.erudit.org/fr/usagers/politique-dutilisation/ 


\section{une cuvre d'amour et de vertu}

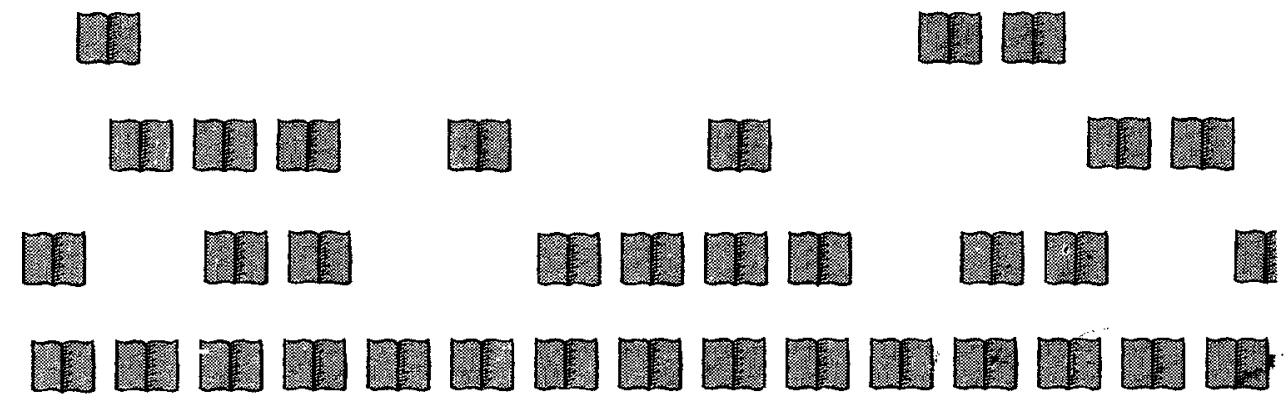

La traduction est un mal nécessaire. Entendons par là qu'elle correspond à une nécessité impérieuse. Les hommes ont besoin de se comprendre entre eux et depuis les jours lointains de Babel, la confusion des langues prévaut dans le monde. L'intervention de l'Esprit-Saint au Cénacle laissait entrevoir une solution heureuse, mais tout indique qu'elle a été sans lendemain...

Il s'ensuit que la traduction est définitivement entrée dans nos mœurs. Surtout dans un pays bilingue et où il est question de biculturalisme. L'expérience a depuis longtemps démontré qu'il est plus facile de traduire des mots que des idées, et des idées qu'un esprit. Le mal n'est sans doute pas très grand aussi longtemps que nous nous en tenons à des textes d'information, à des circulaires administratives, à tout le domaine du pragmatisme. Le traducteur compétent parvient à transmettre dans un autre idiome le renseignement qu'il a mission de communiquer aux destinataires.

Le problème se pose dans des termes entièrement différents dès qu'il s'agit de la création littéraire. Comment en effet pénétrer sans douloureuse effraction dans les arcanes du poète ou du romancier? Le risque n'est-il pas immense de demeurer à la périphérie de son œuvre, de n'en point dégager le mystère ? Nous saisirons l'image sans soupçonner l'émoi qui l'a fait naître, nous cernerons des personnages et une partie de leur drame intérieur nous échappera à jamais.

L'exemple d'un Baudelaire, d'un Mallarmé n'est pertinent qu'au rayon rarement atteint de la perfection. De Baudelaire, son ami Théophile Gautier écrivait que, « possédant à fond la langue anglaise, il débuta par des traductions d'Edgar Poe, traductions tellement excellentes qu'elles semblent des cuvres originales et que la pensée de l'auteur gagne à passer d'un idiome dans l'autre ». Cela est vrai et ne prouve pas grand-chose, sauf qu'il arrive exceptionnellement qu'un écrivain aussi grand dans sa propre langue que son modèle se livre à un travail de traduction qu'il porte à un sommet tel qu'il s'égale à l'original.

Il vaut mieux redescendre de ces hauteurs. Il n'y a qu'un Ruskin qui trouve son Proust! Dans la vie de tous les jours, nous devons nous rabattre sur des 
traductions honnêtes, consciencieuses, d'une fidélité qui se veut exemoinlaire. Le vin est bon, bien qu'une part de son bouquet se soit évaporé. Nous aurions tort de récriminer. Encore heureux sommes-nous en ce pays où tout Canadien cultivé obtient naturellement accès, sans intermédiaire, aux deux plus riches cultures littéraires de l'Occident. Quand il s'agit des écrivains russes, allemands, espagnols ou italiens, nous devons nous en remettre à des serviteurs obscurs qui marchent sur les traces de Jérôme, leur saint patron...

Quel labeur à la fois titanesque et minutieux! Pour exprimer tout l'effort intelligent et sensible qu'il exige, Valery Larbaud, lui-même rompu aux méandres du génie espagnol, a recours à une image suggestive: «... tout le travail de la Traduction est une pesée de mots. Dans l'un des plateaux nous déposons l'un après l'autre les mots de l'auteur, et dans l'autre nous essayons tour à tour un nombre indéterminé de mots appartenant à la langue dans laquelle nous traduisons cet auteur, et nous attendrons l'instant où les plateaux seront en équilibre. »

Peut-être sans s'en douter, Larbaud reprend en des termes très voisins une idée de Victor Hugo, le père d'un des premiers traducteurs français de Shakespeare: «Le traducteur est un peseur perpétuel d'acceptions. Pas de balance plus délicate que celle où l'on met en équilibre des synonymes. L'étroit lien de l'idée et du mot se manifeste dans ces comparaisons des langages humains. »

Le synonyme, voilà l'ennemi - ou le faux ami. Pas la couleur, mais la nuance. Il y faut l'oreille délicate qui sache percevoir les moindres pulsations de la phrase rythmée à la cadence secrète d'un cœur, un œil attentif qui excelle à dégager les rapprochements les plus inattendus entre les idées les plus subtiles. "Traduire, qui est de reconstituer au plus près l'effet d'une certaine cause», comme s'exprime Valéry. À ce niveau, la traduction devient œuvre d'amour et de vertu.

On me permettra une petite anecdote qui ne relève pas de la littérature et qui contribue toutefois à souligner la difficulté qu'éprouvent les hommes à communiquer entre eux. C'était au printemps tragique de 1940. Au moment de la chute de la France, le ministre - il n'y avait pas encore d'ambassadeur - se rend auprès de notre premier ministre en vue d'examiner la situation. L'inconvénient, c'est que M. Ristelhueber ne connaissait pas l'anglais et que M. Mackenzie King ne s'exprimait pas en français. Qu'à cela ne tienne: c'est en allemand, la langue de l'ennemi, que les deux hommes pleurèrent de conserve sur les malheurs de la France.

Roger DUHAMEL, m.s.r.c., de 1'Académie canadienne-française. 